\title{
PARAMETRIC MODELING OF TRANSVERSE PHASE SPACE OF AN RF PHOTOINJECTOR*
}

\author{
B. Sayyar-Rodsari ${ }^{\dagger}$, C. Schweiger, E. Hartman, Pavilion Technologies, Inc., Austin, TX 78759, USA \\ J. Schmerge, M. Lee, P. Lui, E. Paterson SLAC, Menlo Park, CA 94025, USA
}

\section{Abstract}

High brightness electron beam sources such as rf photoinjectors as proposed for SASE FELs must consistently produce the desired beam quality. We report the results of a study in which a combined neural network (NN) and firstprinciples (FP) model is used to model the transverse phase space of the beam as a function of quadrupole strength, while beam charge, solenoid field, accelerator gradient, and linac voltage and phase are kept constant. The parametric transport matrix between the exit of the linac section and the spectrometer screen constitutes the FP component of the combined model. The NN block provides the parameters of the transport matrix as functions of quad current. Using real data from SLAC Gun Test Facility, we will highlight the significance of the constrained training of the NN block and show that the phase space of the beam is accurately modeled by the combined NN and FP model, while variations of beam matrix parameters with the quad current are correctly captured. We plan to extend the combined model in the future to capture the effects of variations in beam charge, solenoid field, and accelerator voltage and phase.

\section{INTRODUCTION}

High brightness electron beam sources such as rf photoinjectors as proposed for SASE FELs must consistently produce the desired beam quality. Free Electron Laser (FEL) applications have been the primary driving force behind this requirement. To achieve the desired beam quality, there have been systematic studies of both the transverse and longitudinal beam properties from the RF photocathode gun at SLAC Gun Test Facility (GTF) [1, 2]. One main objective of these studies has been to identify the factors affecting beam quality and to establish systematic methodologies for minimizing beam emittance. Reliable models that are suitable for emittance minimization are therefore of primary interest $[1,3]$.

Model-based optimization and control has found widespread applications in all aspects of modern life from robotics, aerospace, transportation, materials and process industry, to biology and medicine [4]. For complex applications such as emittance minimization in a photo-injector, however, model-based optimization and control will be viable only if the models are both accurate and computationally efficient.

\footnotetext{
* Work supported by DOE STTR Grant DE-FG02-04ER86225 and DOE-Stanford contract DE-AC02-76SF00515.

† bijan@pavtech.com
}

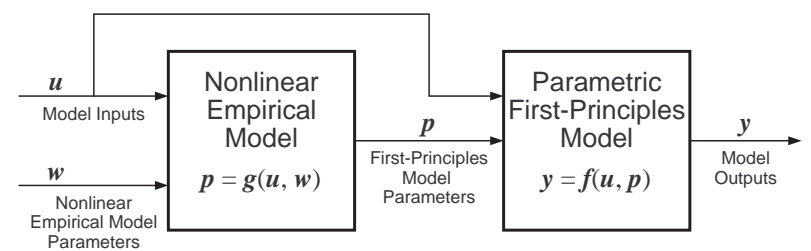

Figure 1: Block diagram of a PUNDA model. The PUNDA model is formed by the series connection of a Nonlinear Empirical Model (NEM) block and a Parametric Firstprinciples Model (PFM) block.

A framework for building accurate and computationally efficient models is shown in Figure 1. This framework, known as Parametric Universal Nonlinear Dynamics Approximator (PUNDA), consists of a series connection of a Nonlinear Empirical Model (NEM) block and a Parametric First-principles Model (PFM) block. The parameters, $\vec{P}$, in the PFM block may vary as a function of process inputs, $\vec{u}$.

For the SLAC GTF beamline ${ }^{1}$, shown in Figure 2, the PFM block is a static nonlinear model, Eq. (1), relating the beam matrix at the exit of the linac to the beam matrix at spectrometer screen. The parameters of the PFM block are the elements of the transport matrix (i.e. $A_{T}, B_{T}, C_{T}$, and $D_{T}$ ) that are functions of the quadrupole current. Note that the beam matrix at the linac exit is an unknown input to the PFM block. The goal of the modeling exercise is to use the measurements of the beam size at the spectrometer screen to systematically identify the parameters of the PFM block (i.e. transport matrix elements), while also estimating the unknown input vector to the PFM block.

\section{PUNDA MODEL FOR TRANSVERSE PHASE-SPACE}

The schematic diagram for transverse phase space measurements at the SLAC GTF is shown in Figure 2. The measurement technique, known as quadrupole scan [2], measures the transverse size of the beam as a function of quadrupole strength. The purpose of the analysis is to identify the transverse beam matrix at the quadrupole doublet $[1,5]$.

\footnotetext{
${ }^{1}$ The SLAC GTF beamline consists of a 1.6 cell S-band gun of the BNL/SLAC/UCLA design followed by a 3 meter linac section. The drive laser is a Nd:Glass CPA laser with a regenerative amplifier that provides 2 ps (fwhm) gaussian UV pulses to the cathode with an approximately uniform, $2 \mathrm{~mm}$ transverse profile.
} 


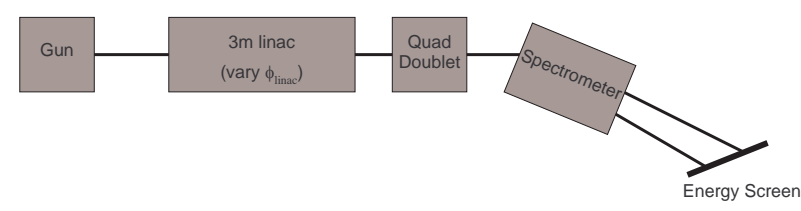

Figure 2: The block diagram for longitudinal and transverse beam measurements at SLAC GTF.

\section{GTF Transport Matrix from Linac Exit to Spectrometer}

The transport matrix for transverse motion of the beam from the exit of Linac to spectrometer is modeled using the first order matrix presented in TRANSPORT [6]. The beam parameters used in constructing transport matrix models are described in [7]. We have used this transport matrix as the first-principles model for the training of the PUNDA models as will be described later.

Designating the transport matrix as $\mathbb{T}=\left[\begin{array}{cc}A_{T} & B_{T} \\ C_{T} & D_{T}\end{array}\right]$, the beam matrix at the quadrupole doublet may be related to the beam matrix at the spectrometer screen as follows:

$$
\left[\begin{array}{ll}
\sigma_{11}^{s} & \sigma_{12}^{s} \\
\sigma_{21}^{s} & \sigma_{22}^{s}
\end{array}\right]=\mathbb{T}\left[\begin{array}{ll}
\sigma_{11}^{e} & \sigma_{12}^{e} \\
\sigma_{21}^{e} & \sigma_{22}^{e}
\end{array}\right] \mathbb{T}^{\prime}
$$

where the superscript ' $\mathrm{s}$ ' indicates the beam matrix parameters at the spectrometer screen while the superscript ' $\mathrm{e}$ ' indicates the parameters at the doublet. The elements of the transport matrix $\mathbb{T}$ are functions of quad current, $I_{2}{ }^{2}$.

\section{SIMULATION RESULTS}

The PUNDA model structure in Figure 1 is used to analyze the transverse phase-space for the beamline of Fig. 2 under a variety of simulation scenarios.

\section{Analysis with Fully Known Transport Matrix}

Using the transport matrix computed in [7] (based on TRANSPORT model), a constrained optimization problem is solved to determine the optimal value of the beam matrix at the quadrupole doublet, i.e. $\sigma_{11}^{e}, \sigma_{12}^{e}$, and $\sigma_{22}^{e}$ given the measurements of the horizontal beam size at the spectrometer screen, $\sigma_{11}^{s}$. Figure 3 shows the measured beam size versus the modeled beam size. The identified beam matrix at the input of the PFM block is consistent with the results reported in [7].

\section{Analysis with Unknown Transport Matrix}

For the second test, we used a neural network for the NEM block in Fig. 1 to estimate $A_{T}$ and $B_{T}$ (as functions of quad current $I_{2}$ ) simultaneously with the elements of the beam matrix at the exit of the linac given measurements of the beam size at the spectrometer screen. From the FP model in Eq. (1), the beam size at the spectrometer screen,

\footnotetext{
${ }^{2}$ This nonlinear function is experimentally determined to be $k_{2}\left(I_{2}\right)=$ $\left(\frac{\alpha I_{2}+\beta}{30.50}\right)^{0.5}$ with $\alpha$, and $\beta$ as tuning parameters.
}

$(\Delta X)^{2}=\sigma_{11}^{s}$, can be described as:

$$
(\Delta X)^{2}=A_{T}^{2} \sigma_{11}^{e}+2 A_{T} B_{T} \sigma_{12}^{e}+B_{T}^{2} \sigma_{22}^{e}
$$

The training of the PUNDA model in this case, involves a systematic search for the weights and biases in the NN model along with the elements of the beam matrix at the quad doublet, i.e. $\sigma_{11}^{e}, \sigma_{12}^{e}$, and $\sigma_{22}^{e}$, to solve an optimization problem that minimizes the error between predicted and measured beam size at the spectrometer screen. Figure 4 compares the PUNDA model prediction of beam size against the measured beam size. Figure 5 compares the identified $A_{T}$ to that computed from first-principles models. It is important to point out that:

1. The results presented here are obtained using only the following constraints, for $I_{2} \in[0, \cdots, 3.9]$, extracted from FP model for beam matrix:

$$
\frac{\partial A_{T}}{\partial I_{2}} \leq 0, \text { and } \frac{\partial B_{T}}{\partial I_{2}} \leq 0
$$

2. With only 14 data points available for the training of the PUNDA model, a better fit to the beam size measurements is obtained compared to that in Figure 3.

\section{Analysis with Partially-known Transport Matrix}

For the third test, we assumed that the only unknown components of the transport matrix are the parameters of the $I_{2}-k_{2}$ relationship of the quadrupole magnet that is defined as $k_{2}\left(I_{2}\right)=\left(\frac{\alpha I_{2}+\beta}{30.50}\right)^{0.5}$. In this case, $\alpha$ and $\beta$ are the outputs of the NEM block, and the elements of the beam transport matrix, i.e. $A_{T}, B_{T}, C_{T}$ and $D_{T}$ are expressed as explicit functions of these parameters (see [7]). The training of the PUNDA model in this case involves simultaneous identification of $\alpha$ and $\beta$ along with the elements of the beam matrix $\sigma_{11}^{e}, \sigma_{12}^{e}$, and $\sigma_{22}^{e}$ given the measurements of the beam size at the spectrometer screen. Figures 6 and 7 demonstrate the prediction results by the PUNDA model in this case. Note that the identified $A_{T}$ (shown in Figures 7 is more consistent with the FP model of the transport matrix (compared to Figure 5). This example underscores the ability of the PUNDA framework to optimally utilize empirical data and FP knowledge/model.

\section{CONCLUSIONS}

We would like to conclude this section with two main observations regarding the applicability of the PUNDA models in GTF applications. First, PUNDA structure offers a framework in which both beam data and first principles models may be used to complement one another. Furthermore, the measured data may be used to fine tune the FP model. Second, a trained PUNDA model may be systematically used to find optimal operation conditions for the GTF. For our first test case, for example, the PUNDA model may be used to find the optimal quad current for which a desired $\left(\Delta X, \Delta X^{\prime}\right)$ may be achieved. We plan to extend the PUNDA modeling methodology to analyze the longitudinal phase space in an rf photoinjector. 


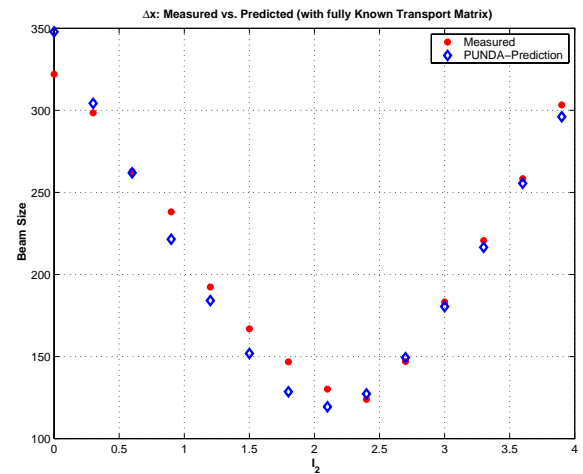

Figure 3: Comparison of measured beam size vs. the beam size predicted by the PUNDA model when the transport matrix was completely determined by TRANSPORT model.

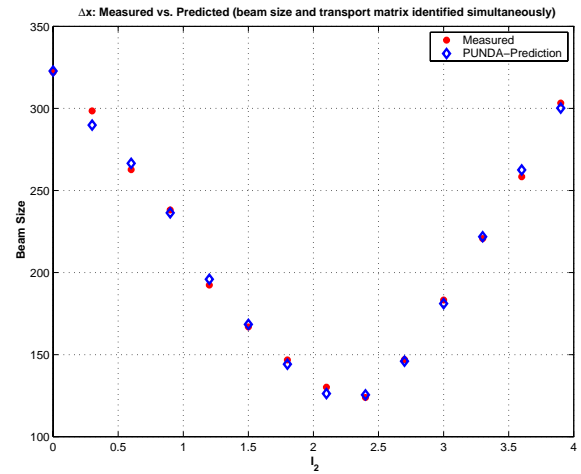

Figure 4: Comparison of measured and predicted beam size when $\Delta X, A_{T}$, and $B_{T}$ are identified simultaneously.

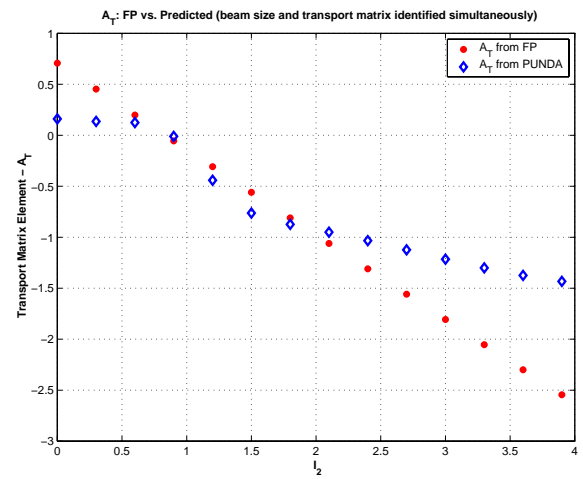

Figure 5: Comparison of identified values for $A_{T}$ vs. the $A_{T}$ values generated by the FP model. While the PUNDA model accurately captures the beam size, as shown in Figure 4, the model for transport matrix parameters deviates from FP values in the absence of additional constraints.

\section{REFERENCES}

[1] D. Dowell, P. Bolton, J. Clendenin, P. Emma, S. Gierman, C. Limborg, B. Murphy, and J. Schmerge, "Longitudinal Measurements at the SLAC Gun Test Facility," SLAC-PUB9541, September 2002.

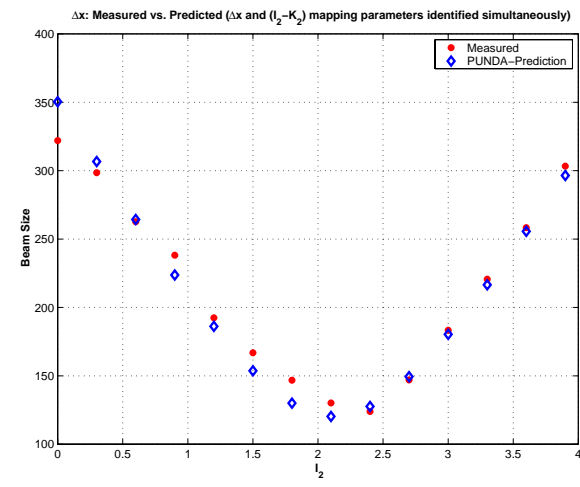

Figure 6: Comparison of measured beam size vs. the beam size predicted by the PUNDA model for the case where beam matrix parameters and $\alpha$ and $\beta$ in $I_{2}-K_{2}$ mapping are identified simultaneously.

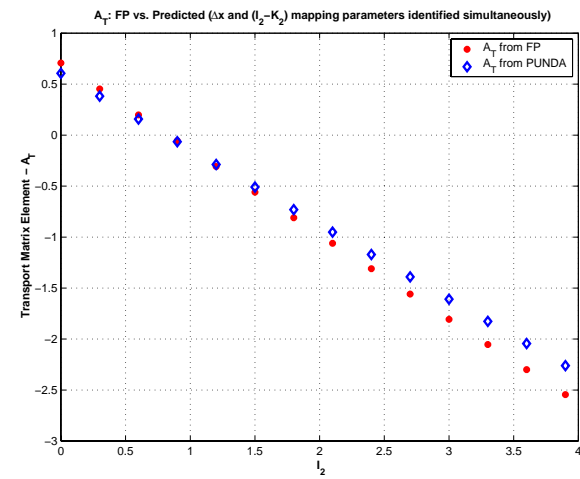

Figure 7: Comparison of identified values for $A_{T}$ vs. the $A_{T}$ values generated by the FP model for the case discussed in Figure 6. The inclusion of the FP knowledge regarding transport matrix in the training of the PUNDA model has made the model for transport matrix elements more consistent with FP information.

[2] D. Dowell, P. Bolton, J. Clendenin, S. Gierman, C. Limborg, B. Murphy, and J. Schmerge, "Analysis of Slice Emittance Measurements For the SLAC Gun Test Facility," SLAC-PUB10727, May 2003.

[3] J. Schmerge, J. Castro, J. Clendenin, D. Dowell, S. Gierman, and R. Hettel, "Emittance and Quantum Efficiency Measurements from a 1.6 cell S-Band Photocathode RF Gun with $\mathrm{Mg}$ Cathode," SLAC-PUB-10763, September 2004.

[4] W. Dunbar and R. Murray, "Model Predictive Control of Coordinated Multi-Vehicle Formations," Conference on Decision and Control, 2002.

[5] J. Schmerge, P. Bolton, J. Clendenin, D. Dowell, S. Gierman, C. Limborg, and B. Murphy, "6D Phase Space Measurements at the SLAC Gun Test Facility," SLAC-PUB-9681, March 2003

[6] K. Brown, F. Rothacker, D. Carey, and C. Iselin, "TRANSPORT: A Computer Program for Designing Charged Particle Beam Transport Systems," SLAC-91, Rev 2, May 1977.

[7] J. Schmerge, J. Clendenin, D. Dowell, and S. Gierman, "GTF Transverse and Longitudinal Emittance Data Analysis Technique," LCLS-TN-05-19, July 2005. 